\title{
Modern materials strengthening techniques by extreme effects
}

\author{
Alexander M. Glezer ${ }^{1,2, *}$ \\ ${ }^{1}$ National Research Technological University "MISiS", Moscow; \\ ${ }^{2}$ Ferrous Metallurgy Central Research Institute named after I.P. Bardin, Moscow
}

\begin{abstract}
In a brief review, the techniques of extreme effects on metallic solids are systematically examined in order to create margin structural states and unique physical and mechanical properties. The principles of complex extreme effects and the engineering of grain boundaries are considered, making it possible to obtain promising multifunctional materials with strength close to the theoretical limit.
\end{abstract}

\section{Introduction}

Modern technological advancement presents ever increasing demands on structural and functional metallic materials. Qualitatively new among them is the requirement on multifunctionality, that is, combining in one and the same material simultaneously high, often difficult to combine mechanical, physical and chemical properties [1]. The existing standard material science principles for the structure and properties formation, based on traditional thermal and deformation effects on a solid, are no longer capable of meeting these requirements, although in some cases the use of expensive multicomponent systems can sometimes deliver the desired results [2].

Of course, the key to obtaining new unusual properties of materials is the creation in them of fundamentally new, previously unknown structural states, and the way to create such structures is to exert extreme effects on solids [3]. According to the famous English physicist R.U. Kahn materials in ultimate states are the latest achievement of materials science and will actively develop in the future [4]. The limiting, in his opinion, are the states caused by the extreme nature of their handling, and, as a consequence, the unique character of the microstructure formed in this case [4]. Further, we will consider, in our view, the most promising extreme effects.

\footnotetext{
* Corresponding author: a.glezer@mail.ru
} 


\section{Melt quenching}

As the most effective method of extreme handling, we, following R.U. Kahn, will consider quenching from liquid state (QLS), which contains fundamentally new opportunities for obtaining promising materials with a unique combination of properties. At present, several QLS techniques are being used, allowing amorphous and microcrystalline alloys in the form of ribbons (up to $100 \mu \mathrm{m}$ ) and width (up to $300 \mathrm{~mm}$ ) to be obtained in various modifications [5], but the most common among them is the spinning method [6], at which the melt is fed under pressure to a rapidly rotating disc-cooler. As a result, we are dealing with a tape with a thickness of 20 to $100 \mu \mathrm{m}$, which has a structure that depends on the alloy composition and the cooling rate. QLS on a rotating disk-refrigerator is a quick process, depending on many physical and technological parameters. Changing or adjusting these parameters, we have the opportunity to obtain optimal conditions for QLS and to intentionally influence the structural state of hardened materials of a given chemical composition.

As the cooling rate increases, a significant change in the character of the emerging structure takes place. First, there is a noticeable polycrystalline structure refinement, and then, depending on the composition of the alloy, the solubility of the components in the solid solution expands, and metastable crystalline phases can form. Finally, if the cooling rate is very high, due to lack of time, crystallization is completely suppressed, and the shear viscosity of the system continuously and smoothly increases with cooling. In the final form, the atomic structure inherent in the liquid state is removed from thermodynamic equilibrium, and at the glass transition temperature $T_{g}$ is homogeneously frozen [7]. The resulting amorphous state is one of the key factors in the quenching experiments.

In accordance with the kinetic approach to the amorphization process, any metal alloy containing at least more than a few percent of the second component can be amorphized upon reaching a certain high cooling rate [8]. Thus, the propensity to amorphize the alloy with a given chemical composition is characterized by a critical cooling rate $V_{c r}$, which, in relation to the spinning method, corresponds to the critical thickness of the quenched strip $t_{c r}$ [6]. For the ultimate cooling rate of $10^{6} \mathrm{deg} / \mathrm{sec}$, which can be achieved with spinning, the value of $t_{c r}$ for most amorphous systems is, as a rule, up to 70-80 $\mu \mathrm{m}$ [9]. It should be noted that in recent years a number of easily amorphous multicomponent metal systems have been discovered (mainly based on $\mathrm{Pd}-\mathrm{Cu}$, $\mathrm{Ti}-\mathrm{Zr}, \mathrm{Zr}-\mathrm{Cu}, \mathrm{Mg}-\mathrm{Cu}$ ), for which $V_{c r}$ values are so low that they can be obtained in the form of massive rods and ingots [10]. However, the overwhelming majority of amorphous and nanocrystalline alloys having practical applications can be obtained only in the form of a strip several tens of microns thick.

The process of transition from a liquid (amorphous) state to a crystalline one can be regarded as an order-disorder transition [11]. Basically, it can be carried out either in the process of cooling from the melt at a certain rate close to the critical one, or in the case of thermal or deformation effects on the solid amorphous state, which is obtained in turn by the QLS. In this case, the crystallization process takes place under conditions of constant heat input (at a constant or at a continuously increasing temperature), taking into account the additional thermal energy released during crystallization. As a result, in most cases, a structure consisting of two distinct structural components, amorphous and nanocrystalline, arises at a certain stage of thermal or deformation processing in the system [12]. The nature 
of the structure in this case depends to a certain extent on the rate of the liquid crystal, the subsequent heating, as well as the annealing temperature and atmosphere, or on the parameters of the deformation effect.

A completely different morphological type of structure can be realized in the implementation of the early stages of crystallization under conditions of sharp cooling of the melt, at which an effective heat removal from the crystallizing system takes place. Such amorphous - crystalline systems have been studied very little, but the mechanical properties implemented in this case can be attributed to the category of unique (hardness $H V=15$ GPa, strength $\left.\sigma_{p}=4.8 \mathrm{GPa}[13]\right)$.

In principle, under the conditions of QLS depending on the value of $V_{c r}$ and the heat dissipation parameters, it is possible to implement five different scenarios and, accordingly, to obtain five different structures and properties of states:

1. Crystallization of the liquid phase is fully implemented in the process of QLS, and we are dealing with a single-phase or multiphase nano- or submicro-crystalline structure.

2. In the process of the liquid crystal, an amorphous state occurs which, during the subsequent cooling down to the point $T_{g}$, has time to partially or completely crystallize. Another option is possible: crystallization is carried out directly from the melt simultaneously with the transition of other areas of the melt into an amorphous state. As a result, an amorphous-nanocrystalline structure is formed.

3. The metastable amorphous state that is stable in a wide temperature range and is characterized by the absence of long-range (crystalline) order in the arrangement of atoms in the presence of a noticeable compositional and topological short-range order in the first coordination spheres is formed in the process of the QLS. Amorphous alloys have high plasticity, and high strength, close to the ultimate strength. Such a combination of mechanical characteristics makes amorphous alloys a unique object, representing both purely scientific and engineering interest [14].

4. Subsequent thermal action according to the appropriate modes results in partial or complete crystallization of the amorphous state obtained by the method of QLS.

5. The subsequent very large deformation effect leads to a partial or complete nanocrystallization of the amorphous state obtained by the QLS techniques.

As we see, crystalline and amorphous structures of the first, second and third types are formed at one stage (QLS), and structures of the fourth and fifth types - in two stages (QLS + thermal or deformation processing). The above structural classification of materials proposed in [15] allows us to physically more reasonably approach the description of the structural features inherent in QLS materials, and as a result, to the purposeful obtaining of the necessary physical and mechanical properties. It should be noted that the nanocrystalline state obtained by QLS is always formed on a "bottom-up" basis (nanocrystals grow from a melt or from an amorphous matrix), which makes quenching from the melt related to the most advanced modern nanotechnologies constructed on the same principle [16] .

This analysis convincingly demonstrates how wide and diverse are the spectrum of the structural states of nanomaterials, which can be obtained in the case of a liquid crystal (amorphous, amorphous-nanocrystalline, and nanostructured). Accordingly, the physical andmechanical properties inherent in these structures are equally diverse and even unique. 
In the process of developing promising amorphous alloys (AA), obtained by the QLS techniques, we solved the following important problems:

- The concept of areas of excess free volume as property damage, responsible for the flow of processes of plastic deformation and destruction of AA [17].

- The nature of the embrittlement fragility of the AS has been established, and methods for targeting effect on the temperature interval of their manifestation [18].

- A method for estimating the temperature and time stability of mechanical properties of AA [19].

- A method for cryogenic processing of AA has been developed, leading to an increase in magnetic and / mechanical properties of AA [20].

- The effect of plasticization upon transition from an amorphous state to a nanocrystalline one [21].

- High-strength AA with carbide hardening have been developed [22].

- A structural model of plastic deformation of AA containing nanocrystals [23].

In the process of development of promising nanocrystalline alloys (NCA) obtained by the QLS techniques, we succeeded in solving the following problems:

- It is shown that QLS can not slow down, but, conversely, can accelerate the flow of phase transformations in a solid body [24].

- The existence of a critical size of nanocrystals, suppressing athermal and thermoelastic martensitic transformations [25].

- The conditions for the production of Finemet-type alloys with unique magnetic and mechanical and mechanical properties [26].

- A new mechanism for the plastic flow of $\mathrm{NCA}$ is proposed: a low-temperature grain boundary microslip [27].

- It is shown that the anomaly of the Hall-Petch dependence in the NCA is due to a change the mechanism of plastic deformation: from dislocation to grain boundary microslip [28].

- A new classification of nanocrystals from the position of their mechanical behavior was suggested[29].

- The structural mechanisms of the destruction of nanocrystals have been established [30].

There is no doubt that the fields of application of amorphous and nanocrystalline alloys obtained by the liquid crystal techniques and having valuable mechanical, magnetic, electrical and corrosion properties will constantly expand.

\section{Megaplastic deformation}

Among the extreme effects, of course, there is the effect on the metallic solid of very large plastic deformations. Recently, interest in this method of impact on materials has increased significantly, since it makes it possible to significantly improve the physical and mechanical properties of metallic materials [31]. To a large extent this is due to the formation of nanostructured states of various types and, in particular, to nanocrystallization processes in the handling of amorphous alloys quenched from a melt [32].

The most common methods of creating very large deformations at the present time are: pressure torsion in a Bridgman chamber (DAC), equal-channel angular pressing (ECAE), 
helical extrusion and stored rolling [33]. The plastic deformations realized in this case are so significant that the usual values of the relative degrees of deformation become senseless, and one should proceed to the true logarithmic deformations of $e$.

Summing up a large number of experimental studies on the structure of materials subjected to MPD, we can state that a complex combination of defect structures containing small-angle and high-angle grain boundaries in a different percentage is observed, as well as defective structures within grains of various degrees of perfection. The threedimensional statistical evaluation of this structure of grains, popular in the literature, gives at best the correlation between high-angle and small-angle boundaries in the material and little information on the nature of those physical processes that occur directly under MPD. In addition, this information, unfortunately, is quite contradictory and ambiguous, since in various experiments the authors observe, as a rule, different structural states on the same materials under similar deformation conditions. In this case, the true nanostructure state $(d$ $<0,1 \mu \mathrm{m}$ ) is not always formed. In steels and alloys, MPD is often accompanied by phase transitions (separation and dissolution of phases, maraging transformation, amorphization) [34]. The latter, as a rule, occurs in intermetallic or multicomponent systems at relatively low temperatures. In our studies, it was possible to increase the value of strength after MPD at room temperature by the ECAE method for high-strength maraging steels by $25 \%$ only due to a substantial fragmentation of products of inverse transformation (particle size about 20-30 nm) while maintaining sufficient ductility [35].

The most coherent concept of large plastic deformations was proposed by V.V. Rybin [36]. On the basis of the concept of the dominant role of the disclination mode in the implementation of large plastic deformations and the fragmentation processes associated with it, it was possible to describe correctly the phenomena occurring at considerable degrees of deformation close to $e=1$. In accordance with the disclination concept, the size of the fragments, the main structural elements, while increasing the deformation, it gradually decreases, reaching a constant minimum value of $0.2 \mu \mathrm{m}$. This essentially means that the transition to the nanostructured state and the size of fragments (grains) of less than $100 \mathrm{~nm}(0.1 \mu \mathrm{m})$ under the action of the disclination mode is hardly possible. In the works of S.A. Firstova et al. [37] draws attention to the fact that the transition to a highly deformed state is accompanied by abrupt changes in the structure of the material that occur at a certain critical value of $e_{c}$. For the case of commercial pure iron $e_{c} \approx 1$. Furthermore, the mechanical behavior of the material changes: the strain hardening instead of the parabolic law for large deformations is governed by a linear law. In a number of studies (for example, [38]) it was shown that with the increase of MPD in the structure, a very large number of excess point defects (mainly vacancies) is formed, capable of stimulating the diffusion phase transitions during deformation.

In [39], we considered the energy aspects of the behavior of a solid under load. Under mechanical effect on a solid body of finite size, a certain amount of deformation energy is pumped into it. The obvious "channel of dissipation" of this energy is plastic deformation. When it is exhausted, another channel can be implemented - mechanical destruction. However, at considerable values of elastic energy, plastic deformation can actually initiate additional "dissipation channels": dynamic recrystallization, phase transformations, and release of thermal energy. In the case of MPD, when the component of the compressive stresses is large, the formation and growth of splitting cracks is partially or completely 
suppressed and, consequently, the implementation of the fracture process is substantially complicated. In other words, using ECAE, DAC or similar loading schemes, we force the solid body to deform without breaking down. If the concept of VV. Rybina [36] is correct, then plastic deformation is effective up to a certain limit corresponding to the formation of a critical defect structure, and then other physical processes should be the main dissipation channels: dynamic recrystallization, phase transformations and / or heat release. In [40], we proposed three possible cases for the development of further events. In the case where the processes of dislocation (disclination) rearrangements are facilitated in the material (for example, in pure metals), low-temperature dynamic recrystallization follows the plastic deformation. The local regions of the structure are "cleared" of defects, and in the new recrystallized grains the process of plastic flow begins again with the help of dislocation and disclination modes. In this case, dynamic recrystallization acts as a powerful additional channel for the dissipation of elastic energy. In the case when the mobility of the plastic deformation carriers is relatively low (for example, in solid solutions or intermetallic metals), a powerful additional channel for the dissipation of elastic energy is the phase transition. More frequently, this is a "crystal-amorphous state" transition. As a result, the plastic flow is localized in an amorphous matrix without the effects of strain hardening and the accumulation of large internal stresses. Apparently, there is an intermediate case in which an additional channel of dissipation can serve as disclinational rearrangements, which lead to the stabilization of the fragmented structure observed in some experiments as the MPD is being developed.

Obviously, the transition from one scenario of structural reorganizations to another one also depends on the parameter $\left(T_{M P D} / T_{m}\right.$, where $T_{M P D}$ is the MPD temperature considering the possible effect of heat release and $T_{\mathrm{m}}$ is the melting point. On the basis of a detailed analysis of the structural processes taking place at the MPD, we formulated three basic principles for the implementation of the MPD: the principle of hydrostaticity of the stress state scheme for MPD, the principle of non-dislocation modes of dissipation of elastic energy during the MPD implementation and the principle of cyclic structural-phase transformations in the MPD process. The essence of the first two principles is clear from the mentioned above. Regarding the principle of cyclic recurrence, its essence is of a synergetic nature under the conditions of an open system, under which there is a constant "injection" of deformation energy in the MPD process [39]. If we consider a specific microvolume of a deformed sample, then, following dynamic recrystallization or amorphization, the process of plastic deformation begins "from scratch" in the newly formed recrystallized grain or in the region of the amorphous phase. Further, in the microvolume under consideration under the action of deforming stresses, defect accumulation takes place again, and the process is repeated. Cyclical structure formation in MPD under conditions of dynamic recrystallization for pure metals was first established by V.A. Likhachev in the paper[41] and then repeatedly confirmed by other researchers. The cyclic recurrence at MPD under conditions of phase transitions "crystal $\Leftrightarrow$ amorphous state" for intermetallic metals was recently established in the paper [42].

The crunch numbers show that at low values of the Peierls barrier and in the presence of an effective dissipation channel, the curve of plastic flow has a cyclic character with a certain "wavelength" $\Delta \varepsilon$ [42]. The fact that we practically never fix such flow curves in the experiment does not counter these numbers. The process of plastic flow at all stages of its 
development proceeds, as it is well known, extremely heterogeneously, and the various regions of the deformed crystal are at various stages of their evolution.

It is important to emphasize that the flow of MPD does not guarantee at all the formation of a nanocrystalline state with a crystallite size of less than $100 \mathrm{~nm}$, separated by high-angle or interphase boundaries. For example, in pure metals with high dislocation mobility it is virtually impossible. An important factor in the formation of nanostructures under MPD is the occurrence of phase transformations of the martensitic and diffusion type, twinning, as well as the transition to the amorphous state. Stimulating phase transformations or twinning by varying the temperature and chemical composition of materials, we are able to obtain nanostructures of various types.

\section{Cryogenic dislocation}

It is known [25] that the martensite transformation is suppressed in nanocrystalline structures. The greater the deformation of the transformation is, the greater is the critical size that suppresses the growth of martensitic crystals [43]. This circumstance does not make it possible to obtain, under standard processing conditions, nano-sized martensite crystals, which can obviously significantly increase the strength and a number of other functional properties of metallic materials. Moreover, low-temperature deformation has been used for a long time, which has a number of significant features both from the point of view of the formation of dislocation structures and from the point of view of activation of martensitic transformations [44]. Therefore, we carried out experiments on the lowtemperature $(77 \mathrm{~K})$ deformation of $\mathrm{Fe}-\mathrm{Cr}-\mathrm{Ni}$ alloys having a single-phase fcc lattice of the $\gamma$-phase at room temperature, which remained stable (it did not convert into a bcc lattice of $\alpha$-martensite, or cooled to $77 \mathrm{~K}$, nor during plastic deformation at room temperature). It turned out that under certain experimental conditions, simultaneous cooling to cryogenic temperatures and plastic deformation (rolling) are capable of stimulating a martensitic $\gamma \Rightarrow \alpha$ transformation, and with a significant amount of $\alpha$ - martensite (a volume fraction of more than 0.6), a significant (almost 3-fold) increase strength and hardness of the chromiumnickel alloy. Small alloying additives A1 contributed to dispersion hardening and an additional increase in mechanical properties during low-temperature annealing by an additional $15 \%[45]$.

Structural analysis showed that such a significant strengthening effect in cryogenic deformation is due to the formation of martensitic nanocrystals of 10-50 nm in size and strongly fragmented austenite crystals with a size of not more than $100 \mathrm{~nm}$, which were preserved at low temperature tempering. Of course, the combined effect of plastic deformation and temperature, when each of these factors is ineffective, can serve as an effective way to create a unique structure and properties of materials and can be successfully implemented for other metal systems.

\section{The combined extreme effects principle}

The structure formation processes discussed above have shown a variety of nanostructured states that can be obtained using extreme effects on the condensed state of materials. The 
method of QLS, MPD and laser action cause the appearance of unusual structures that cause unique physical and mechanical properties. Cryogenic dislocation under certain conditions leads to the formation of martensitic nanostructures with significantly higher strength, ductility and satisfactory corrosion resistance. Ultrasonic effects lead to an appreciable relaxation of internal stresses and to a tangible hardening of the surface layers. We had an idea to combine the above-mentioned extreme effects and "build" them into a single technological chain, which could lead to qualitative changes in the nature of the final structure, and consequently, to the possibility of obtaining unique properties of metallic materials subjected to complex extreme effects. Separate links of this chain were already implemented by us (QLS, leading to an amorphous state, + MPD) [46]. This led to the unique magnetic and mechanical properties of metal-metalloid alloys that could not be obtained earlier [47]. It is also possible to build other technological chains that have not been studied before. For example, MPD + ultrasound or QLS + cryogenic dislocation. Of course, the most interesting is the four-link chain: QLS with the formation of an amorphous, amorphous-nanocrystalline or nanocrystalline structure, + MPD + cryogenic dislocation (or MPD at cryogenic temperatures) + ultrasonic treatment. But for the successful implementation of complex processing it is necessary to "synchronize" the parameters of all methods of effecting the material to obtain qualitatively new structures and structurally sensitive properties. Such "synchronization", of course, should be the subject of further research, which will consistently consider the various options for extreme impacts and optimize the conditions for their implementation. Such a statement of research is absolutely authentic and has not yet been adequately reflected in the scientific literature.

\section{The grain boundaries engineering principle}

The most important element of the materials structure are the grain boundaries. Effecting them, you can control the physical and mechanical characteristics of materials. For example, the formation of grain-boundary segregations of "harmful" impurities can lead to materials brittleness and to a significant strength reduction. On the contrary, the intentional creation of "useful" impurities on the grain boundaries, increasing their cohesive strength, can significantly improve the mechanical properties of materials. By effecting purposefully the structure and phase composition of the boundaries, we are able to control the properties of materials. The grain boundaries engineering principle can be successfully applied, for example, to alloys obtained by the method of QLS or MPD. The promising nature of this principle can be illustrated by the example of a recent study [48]. For nanocrystalline Ti-Ni$\mathrm{Cu}$ alloys, it has been shown that the introduction of grain boundaries of nanoparticles of boride phases Ti2B and TiB2 of about $5 \mathrm{~nm}$ in size by means of the engineering principle suppresses the low-temperature grain-boundary slip process and shifts the anomaly region of the Hall-Petch ratio to smaller values of the average size nanocrystals. This allows a $20 \%$ increase in the maximum normalized value of microhardness and closely (by 95\%) to approach the theoretical limit of this characteristic. 


\section{The conclusion}

We have analyzed the main regularities of structure formation and the formation of unique physical and mechanical properties in metallic materials under extreme effects (quenching from melt, megaplastic and cryogenic dislocation). The principle of combined extreme effects is proposed, on the basis of which multifunctional materials combining high strength with a given level of physical properties were developed. Using the engineering grain boundaries method model alloys with real strength, close to the theoretical limit have been obtained. We have considered only two principal consequences of the above discussion of extreme effects on the structure and properties of metallic materials, but undoubtedly, the interested reader will find for himself other useful ideas for new effective ways of creating promising materials of tomorrow. At least, we really hope so.

The work was carried out in accordance with the state task of the Ministry of Education and Science of the Russian Federation No. 2017/113 (2097).

\section{References}

1. Osipyan Yu. A., Mogutnov B.M., Gleser A.M. Izvestiya RAN, Ser.phis. 661230 (2002)

2. Firstov S.A .et al., In the collection. Modern problems of physical material science. Proceedings of IPM NASU. 18 (Kiev: IPM, 2009) p. 140

3. Fortov V.E . Extreme states of matter (M.: Fizmatlit, 2009)

4. Cahn R. W. The Coming of Materials Science (Pergamon Mater., Sci, 2001) [Kan R.U. Material Science Development (N. Novgorod: N.I .Lobachevsky University Publishing Center, 2011)]

5. Amorphous metal alloys, coll. scientific works (Edited by F.E. Lyuborskii) (Moscow: Metallurgy, 1987)

6. Filonov M.F., Anikin Yu.A., Levin Yu. B. Theoretical foundations for the production of amorphous and nanocrystalline alloys by the method of superfast hardening ( $\mathrm{M}$.: MISiS, 2006)

7. Zolotukhin I.V., Kalinin Yu. Yu. UFN 16075 (1990)

8. Sudzuki K., Fujimori X., Hashimoto K. Amorphous metals. (Moscow: Metallurgy, 1987)

9. Glezer A.M., Potekaev A.I., Cheretaeva A.O. Thermal and time stability of amorphous alloys. (Cambrige: CISP; N-Y .: Taylor \& Francis, 2017).

10. Inoue A. in Amorphous and nanocrystalline materials: preparation, properties and applications (Advances in Materials Research) (Eds A. Inoue, K. Hashimoto) (Berlin, Heidelberg, New York: Springer-Verlag, 2001). p.1

11. Frenkel I. and Introduction to the Theory of Metals (L .: Nauka, 1972)

12. Gleser A.M. Russian Chemical Journal XLVI 557 (2002)

13. Glezer A.M., Shurygina N.A. Amorphous-nanocrystalline alloys. (Moscow: Fizmatlit, 2014).

14. Gleser A.M. et al., Mechanical Behavior of Amorphous Alloys (Novokuznetsk: Izd-vo SibGIU, 2006) 
15. Glezer A .M., Permyakova I.E. Nanocrystals hardened from a melt. (Moscow: Fizmatlit, 2012).

16. Tretyakov Yu. D. Bulletin of the Russian Academy of Sciences 7713 (2007)

17. Glezer A.M., Betekhtin V. I. FTT 4085 (1998)

18. Glezer A .M. J. Phys. IV 8,175 (1998)

19. Glezer A.M. et al. Russian Metal. (Metally) 4,274 (2015) /

20. Zaychenko S.G. The patent of the Russian Federation No. 2154869, 1999.

21. Glezer A.M., Permyakova I .E., Manaenkov S. E. Reports of the Russian Academy of Sciences. 418,181 (2008)

22. Glezer A.M., et al. Patent of the Russian Federation No. 2001457, 1089

23. Glezer A.M. et al. Bulletin Russian Acad. Sci: Physics. 771391 (2013).

24. Gleser A.M. et al., Reports of the Russian Academy of Sciences 407478 (2006)

25. Glezer A. M. et al. J. Nanoparticle Research 5,551 (2003)

26. Shurygin N.A., et al. Proceedings of the Russian Academy of Sciences, ser. phys. 76 52 (2012)

27. Pozdnyakov V. A., Glezer A. M. Nanostruct. Mater. 6,767 (1995)

28. Glezer A.M, Pozdnyakov V.A. ZhTF 2131 (1995)

29. Gleser A.M. Deformation and destruction of materials 21 (2010)

30. Gleser A.M., Pozdnyakov V.A. FTT 47793 (2005)

31. Andreevskii R.A., Glezer A.M.UFN 179337 (2009)

32. Glezer A.M., Kozlov E.V. et al. Plastic deformation of nanostructured materials. (Cambrige: CISP; N-Y .: Taylor \& Francis, 2017).

33. Utyashev F.Z., Raab G.I. Deformational methods for obtaining and processing ultrafine-grained and nanostructured materials. (Ufa: Guillem, 2013).

34. Gleser A.M., Gromov V.E . Nanocrystals created by extreme effects (Novokuznetsk: Izdatelstvo "Inter-Kuzbass" 2010)

35. Edneral A.F., et al. Proceedings of the Russian Academy of Sciences, ser. phys. 69 1350 (2005)

36. Rybin V. V. Large plastic deformation and destruction of metals (M .: Metallurgy, 1986)

37. Verst, S.A., et al., Izv. Universities. Physics 341 (2002)

38. Gapontsev V.L., Kondratjev V. V. Reports of the RAS 385684 (2002)

39. Gleser A.M., Metlov L.S. FTT 521090 (2010).

40. Gleser A.M., Pozdnyakov V.A. Deformation and destruction of materials 49 (2005)

41. Likhachev V.A., et al., FMM 451163 (1978)

42. Glezer A.M., Sundeev R.V., Shalimova A. V. Reports of the RAS 44039 (2011)

43. Glezer A.M., et al. Material Science 123 (2007)

44. Starcev V.I., Ilyichev V.Ya., Pustovalov V.V. Plasticity and strength of metals and alloys at low temperatures. (Metallurgy 1975)

45. Glezer AM et al. Deformation and destruction of materials 1023 (2011)

46. Gleser, A.M.et al., Izv. RAS, ser. phys. 731310 (2009).

47. Glezer A.M., et al. Patent of the Russian Federation No. 2391414, 2008.

48. Glezer A.M., Shurygina N.A. et al. J. Mater. Sci. Technol. (31 1 91, 2015). 